\title{
La paradoja de los sistemas de participación ciudadana y el despertar de las insurgencias en Chile
}

\author{
The paradox of citizen participation systems \\ and the awakening of insurgencies in Chile
}

\section{Christian Andrés Quinteros Flores*}

\section{* Trabajador Social de la Universi- dad de Valparaíso y magíster en Ciencia Política de la Universidad de Chile. Doctorando en Ordena- miento Territorial por la Univer- sidad Nacional de Cuyo, Argen- tina. Es académico de la Escuela de Administración Pública de la Universidad Católica del Maule, Chile, y miembro del Grupo de In- vestigación en Descentralización y Territorio de la Universidad de Chile.}

$\triangle$ cquinteros@ucm.cl https://orcid.org/0000-00026145-9347

\section{RECIBIDO: 9.3.2021} ACEPTAD 0: 26.4.2021

\section{Resumen}

En 2019 la sociedad chilena atravesó una de las mayores crisis sociales y políticas de que se tenga memoria, lo que puso en evidencia los altos niveles de desigualdad social y territorial y propició un proceso de profundos cambios institucionales. Un año después, profundizando esas brechas y ralentizando los cambios, la pandemia apareció como una pausa en las redefiniciones institucionales. Como efectos de ambas crisis, hoy se observan en Chile aumento exponencial de tomas de terrenos, enjuiciamiento y desacreditación del sistema político, cambios en los tradicionales ejes políticos, aumento de los populismos y debates sobre el modelo de desarrollo a adoptar, y, como cuestión transversal, cuestionamientos profundos a las formas tradicionales de participación social en un sistema democrático. Es precisamente en este último aspecto donde se advierte la paradoja que nos ofrece la institucionalidad chilena en materia de participación social. Con un diseño robusto y multicanal de espacios de participación, esta institucionalidad presenta, al mismo tiempo, altos índices de desafección en los sistemas tradicionales, pero un aumento sostenido de las movilizaciones sociales y de la incidencia efectiva de los grupos de activistas. Este ensayo contextualiza desde la perspectiva de la 
gobernanza los nuevos desafíos institucionales en materia de participación ciudadana que debe asumir el país de cara a la redacción de una nueva Constitución política.

Palabras clave: crisis política, instituciones políticas, sociedad civil, Chile.

\section{Abstract}

In 2019, Chilean society went through one of its greatest social and political crises in memory, revealing high levels of social and territorial inequality and fostering a process of profound institutional changes. A year later, deepening those gaps and slowing changes, the pandemic appeared as a pause in institutional redefinitions. As effects of both crises, today the country is experiencing an exponential increase in land seizures, prosecution and discrediting of the political system, changes in the traditional political axes, increase in populism and debates on the development model to be adopted, and, as a cross-cutting issue, profound questions to the traditional forms of social participation in a democratic system. It is precisely in this last aspect that the paradox offered by Chilean institutions in terms of social participation is noticed. With a robust and multichannel design of participation spaces, the institutionality presents at the same time, high rates of disaffection in traditional systems but a sustained increase in social upheaval and the effective incidence of activist groups. This essay contextualizes, from the perspective of governance, the new institutional challenges in terms of citizen participation that the country must take on when drafting a new Political Constitution.

Keywords: political crises, political institutions, civil society, Chile.

\section{Introducción}

Las crisis sociales y políticas siempre obligan a la creación de nuevos diseños institucionales. En el caso de la participación ciudadana a escala mundial, ciertos fenómenos la han condicionado: el Estado-nación se ha transformado en un Estado red que está obligado a compartir el poder, ya sea con los gobiernos subnacionales o con la sociedad civil (Castells, 2005), y la democracia representativa ha entrado en una profunda crisis (Svirsky, 2010), amenazados por el despertar de movimientos insurgentes (Svirsky, 2010; Miraftab, 2018). De esta manera, activistas buscan por fuera lo que no pueden conseguir por dentro del sistema.

Si bien en Chile han existido grupos activistas como los pobladores ilegales (Castillo, 2014; Murphy, 2014), hoy se advierte un aumento de grupos que desafían los requisitos institucionales para satisfacer sus demandas: desde grupos de pobladores que no están dispuestos a seguir esperando mientras cumplen con los exigentes requisitos de ahorro que establece la política pública de vivienda, hasta pobladores que no asisten a 
las sesiones de difusión de un nuevo instrumento de ordenamiento territorial por autoevaluar la inocuidad de su acción o porque sencillamente perciben que son instrumentalizados. Son señales inequívocas de que la democracia directa busca transformarse hoy en una verdadera opción de incidencia pública, desestimando la de tipo representativo. Como señala Svarsky (2010): «Una tarea importante de nuevas máquinas de guerra activistas es, entonces, escapar de la trampa dentro del agujero negro del discurso mayoritario sobre la sociedad civil, capturada y definida por las nociones generalizadas de "participación representativa"».

Chile, pese a la alta desafección política, se ubica en el segundo lugar en Latinoamérica con mejor calidad de democracia y en el decimoséptimo en el mundo, con bajo puntaje solo en participación política: 6,67 de un total de 10 puntos. En las otras dimensiones de esta medición, como procesos electorales y pluralismo, funcionamiento de gobierno, cultura política y libertades civiles, se ubica por sobre el promedio de la región (The Economist Intelligence Unit Limited, 2021). A modo de hipótesis, este parámetro pone en alerta la situación en otros países y sociedades de la región, ya que desdibuja los reales parámetros de la participación política, considerando que en Chile sigue siendo débil en términos de incidencia. Algo similar ocurre en materia de participación ciudadana, donde la incidencia de la denominada participación de la sociedad civil es cada vez menor, aun cuando ha aumentado el número de organizaciones sociales. En Chile, las organizaciones de la sociedad civil (OSC) han sido un actor clave desde 1990. En los últimos cinco años se han constituido más de 85.000 nuevas OSC. En promedio se crean más de 17.000 por año y más de 1400 cada mes (Irarrázaval y Streeter, 2020).

\section{El problema de la gobernanza}

La palabra gobernanza generalmente se utiliza con una connotación positiva y, casi intuitivamente, se equipara a una virtud o fortaleza de las organizaciones públicas. Se distancia del concepto de gobernabilidad, que solo refiere a mantener el control político y el orden institucional. Es común que la gobernanza sea descrita como una meta de política pública o como un distintivo de las prácticas propias de organizaciones modernas y democráticas, apuntando a procesos como la participación ciudadana, la transparencia, el control interno, la rendición de cuentas, la gestión basada en resultados o la integridad, entre otros aspectos (Portal, 2016). Claramente, entonces, el problema de la participación social, en sus expresiones electorales de una democracia representativa o ciudadana, es un aspecto clave en la consolidación de la gobernanza y debe abrirse a la discusión de su efectividad y legitimidad social.

Una de las interrogantes que se presentan en este análisis es si hoy las políticas públicas y sus mecanismos de participación ciudadana están siendo lo suficientemente 
democráticos y participativos, y, más aún, si están siendo eficientes para captar las demandas de la comunidad.

Según Maya et al. (2019), durante las últimas décadas, el papel de las administraciones gubernamentales ha tendido a ser cada vez más objeto de observación, de reclamo y de cuestionamientos, que en algunos casos derivan de su incapacidad para responder eficientemente a las demandas de la sociedad y aparecen como producto del agotamiento de los esquemas tradicionales de gobernar y administrar el territorio. En este contexto, la gobernanza surge como modelo alternativo para resolver las deficiencias que hoy afectan a los Estados de la región, ya sea por el acelerado crecimiento urbano y demográfico, por los efectos del agotamiento de los recursos naturales, por el incremento de los índices de contaminación, por el aumento de la segregación territorial (Arriagada, 2017) o por el distanciamiento entre los ciudadanos y las autoridades, entre muchos otros factores.

El problema de la gobernanza incluso ha difuminado los límites jurisdiccionales de lo territorial y se ha traslado a una dimensión multinivel que no solo afecta a lo local. Mariano Ferrero (2010, p. 121) afirma que la complejidad de los asuntos mundiales ha alentado que las actividades económicas se vean influidas por agentes institucionales de niveles territoriales múltiples, que interactúan en términos cooperativos y no jerárquicos. La noción de gobernanza multinivel se refiere a procesos de «intercambio negociado y no jerárquico entre instituciones de nivel transnacional, nacional, regional y local, e incluso puede extenderse a procesos de gobernanza público-privada» que deben acordar sobre las fronteras de los problemas (Comisión Económica para América Latina y el Caribe [CEPAL], 2016).

Desde esta perspectiva, la gobernanza requiere ponerse de acuerdo sobre las reglas básicas para una sana convivencia social. Para Sánchez (2017), se trata del conjunto de mecanismos, procesos y reglas a través del cual se ejerce la autoridad sobre la gestión de los recursos naturales, las conductas entre las partes, la protección de la naturaleza y de los derechos básicos, y se relaciona con el funcionamiento de las instituciones y las reglas que influyen sobre él, con miras al presente y al futuro, apuntando de esta manera a un enfoque de sostenibilidad.

Iracheta Cenecorta e Iracheta Carroll (2014) señalan que la gobernanza se entiende como el proceso de irrupción social en la cosa pública; es decir, la construcción de nuevas capacidades que hacen de las organizaciones de la sociedad actores que se involucran en los procesos antes delegados exclusivamente al gobierno. La gobernanza debe incluir la gobernabilidad, pero esta no explica a la primera. La ciudad y la región son los espacios más importantes de la gobernanza; en ellas se crean las condiciones del desarrollo y las reglas de la comunidad. Como consecuencia, la acción pública ocurre por la coordinación de la acción colectiva y no por la subordinación de la ciudadanía al aparato gubernamental. Esto es así porque se reconoce que el Estado no lo puede hacer 
todo. Su papel relevante es coordinar la acción pública con la participación de los actores sociales. Esta coordinación deja de ser vertical para convertirse en una red de relaciones horizontales o, en su mejor versión, en el marco de un sistema de políticas públicas tipo bottom up. ${ }^{1}$

Una de los factores que desencadenaron la crisis política de 2019 en Chile es precisamente la desconfianza de la ciudadanía ante las reglas del juego y las formas de decidir que había adoptado el Estado-nación en los últimos 30 años, incluidas las formas de estimular la participación ciudadana en los asuntos públicos. Sin embargo, la concepción de participación ciudadana supone el desarrollo previo de una base epistemológica y ontológica que es necesario definir para comprender la paradoja de este ensayo, que refiere a la emergencia de movimientos insurgentes y activistas en un sistema institucional que cuenta con nutridas normas de participación. El punto de partida es que la participación ciudadana se inscribe en un contexto de diseño del Estado-nación que busca incidir en asuntos públicos simplemente invitando a la ciudadanía a decidir, para legitimar la decisión $x$ o y. Por ello, el análisis sobre la calidad de este proceso y su real incidencia merecen un tratamiento especial.

El tema de la participación ciudadana debe analizarse en el marco del sistema de gobierno en que se pretende impulsar. Por un lado, en un sistema de democracia representativa, los mecanismos de participación ciudadana serán distintos que en una democracia directa. La desconfianza social que ha impactado a los países de la región ha debilitado consecuentemente la calidad de la representación política. Para autores como Svirsky (2010), la participación «representativa» es problemática por al menos dos razones. En primer lugar, la participación en el proceso político formal de «representación en democracia» no implica en sí misma necesariamente una actitud crítica o acción, sino que busca una vida menos represiva y más creativa. En segundo lugar, la pasividad no es necesariamente un signo de anemia política, sino que puede ser una expresión cultural. La ausencia de una participación masiva visible podría ser un signo de cumplimiento inconsciente y preconsciente de las formas en curso de la opresión, y puede tener un impacto más enérgico en la perpetuación de un régimen (Svirsky, 2010).

En definitiva, las posibilidades de participación corren hoy por dos cuerdas distintas: por un lado, los sistemas tradicionales de participación ciudadana y, por otro, las reacciones institucionales a movimientos que van por fuera y buscan incidir directa y urgentemente por otras vías, casi siempre apartadas de lo institucional. 


\section{La situación en Chile}

Esta tensión entre las formas de participación ciudadana en un sistema de democracia representativa o en un sistema directo podría ayudar a explicar la paradoja de la alta densidad de normas favorables a la participación y los bajos índices de afección e incidencia pública que caracterizan al actual sistema de participación social y ciudadana en Chile.

Figura 1. Institucionalidad de la participación ciudadana en Chile

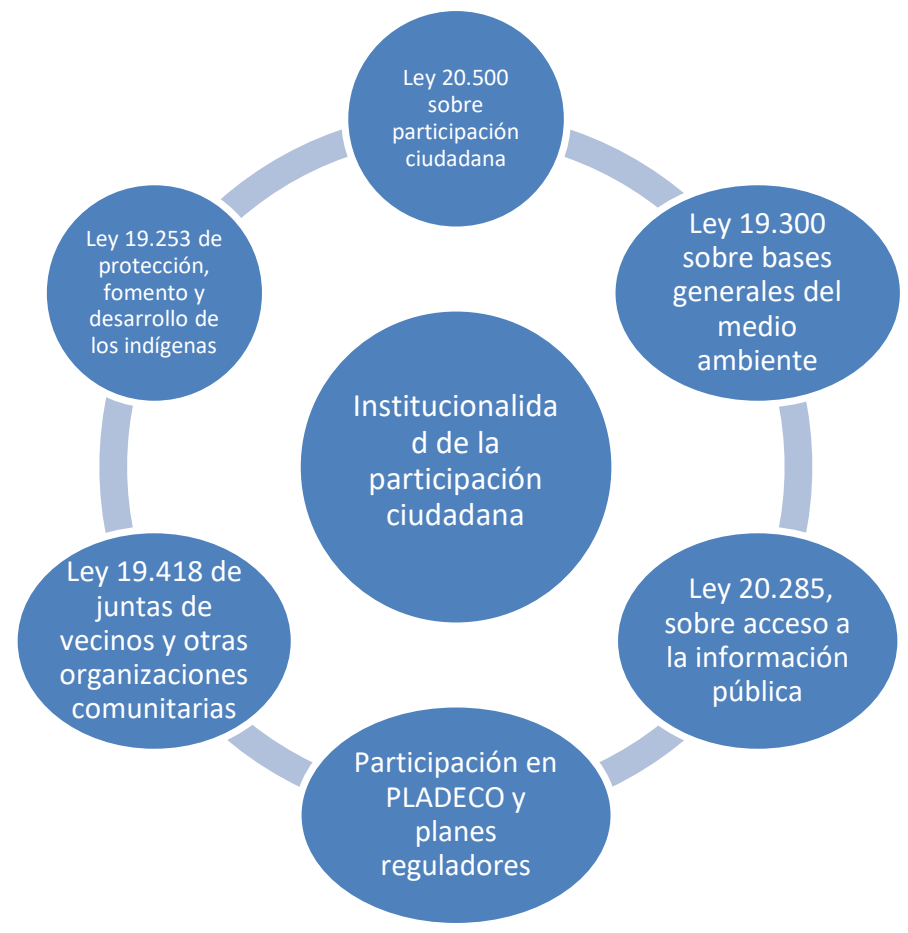

Fuente: Elaboración propia basada en la revisión de las leyes 19.253, 19.418, 18.695, $19.300,20.285,20.500$ y DFL 458, Ley General de Urbanismo y Construcciones (Chile, 1993, 1997, 2006, 1994, 2008, 2011, 1976).

La figura 1 evidencia la alta densidad de normas jurídicas disponibles en Chile que permiten - al menos teóricamente - la incidencia ciudadana en los proyectos públicos. Desde la posibilidad de integrar consejos asesores de los órganos de gobierno, pasando por la oportunidad de incidir en la aprobación de proyectos con impacto ambiental, hasta la posibilidad de acceder a la información pública en forma oportuna y veraz, se 
trata de mecanismos que el Estado chileno pone a disposición para canalizar las demandas ciudadanas en los territorios. Además, este impulso a la descentralización de las decisiones se ha acrecentado desde 2018 con las modificaciones a las normas de regionalización y de descentralización político-administrativa promulgadas a través de la ley 21.073 (Chile, 2018a), que permitirá que este año 2021 por primera vez se produzca la elección popular de gobernadores regionales, y de la ley 21.074 (Chile, 2018b), que introduce en la gestión regional la creación del Plan Regional de Ordenamiento Territorial (PROT) y de nuevas áreas metropolitanas para una mejor gobernanza territorial.

Sin embargo, la utilización de los mecanismos participativos a escala local-comunal sigue siendo débil y precaria. A pesar de los avances en la institucionalidad formal y en la implementación de las leyes de transparencia, acceso a la información y participación ciudadana, ya señaladas, la situación de la gobernanza local en Chile aún se encuentra lejos del ideal (Hernández, 2020). Según Hernández, se observa que tanto los espacios institucionales de transparencia como los de participación son externos al municipio, con lineamientos marcados por la legislación nacional, algo que evita su adaptación a los diversos entornos locales. Se aprecia en definitiva una extraordinaria rigidez normativa en estos mecanismos.

Además, en el ámbito de la planificación territorial, la Política Nacional de Desarrollo Urbano 2014 de Chile declara como un objetivo la participación ciudadana «efectiva». Sin embargo, la participación de la población en asociaciones de este tipo ha decrecido significativamente en general.

Las estadísticas sobre participación ciudadana contenidas en las encuestas CASEN 2000 y CASEN 2009, y en la Encuesta «Santiago Cómo Vamos 2013» permiten concluir que la participación en organizaciones sociales disminuyó casi a la mitad en la ciudad de Santiago en los últimos trece años y que, en general, no existe interés por parte de la ciudadanía en instrumentos de planificación participativa. Se identifican las implicaciones que tiene esta prescindencia en la construcción de una política de planificación urbana más participativa, y los desafíos que tal situación plantea. (Herrmann y Van Klaveren, 2016, p. 175)

Herrmann y Van Klaveren (2016) resaltan la falta de interés y la desconfianza de la ciudadanía hacia instrumentos que le permitirían una participación más activa en la definición de políticas urbanas.

En la ciudad de Santiago la población prefiere convocar a un grupo de expertos que llamar a un plebiscito como mecanismo de toma de decisión, y 75\% de los santiaguinos declara que no participaría en un presupuesto participativo comunal. 
En el ámbito electoral, también se advierte en Chile que la disminución en la participación electoral ha sido sistemática desde principios de la década de 1990 (Programa de las Naciones Unidas para el Desarrollo [PNUD], 2017). Explicarían esta baja gobernanza democrática local la baja participación ciudadana, el papel en ocasiones monopólico del capital político por parte de dirigentes sociales territoriales, la desarticulación que se presenta con las bases sociales y la percepción de inoperancia de los mecanismos institucionales de participación (Hernández, 2020). Si bien a simple vista se aprecia en la figura 1 una robusta institucionalidad en materia de posibilidad de incidencia pública, parecería que no es el camino del análisis que se debiera llevar para contextualizar y comprender cabalmente la emergencia de nuevos movimientos insurgentes.

\section{La irrupción de los insurgentes}

Pertinente es preguntarse, entonces: ¿Qué explica la crisis de participación en los sistemas tradicionales y la irrupción de movimientos rupturistas que buscan nuevas formas de incidencia en las políticas públicas? ¿Cuáles son las razones del surgimiento de nuevos movimientos sociales que no utilizan estos mecanismos? ¿0 es simplemente porque están cansados de utilizarlos toda vez que no son vinculantes con las decisiones finales que se adoptan en sus territorios?

La tarea de indagar en las posibles razones del aumento de formas insurgentes de participación social en Chile requiere considerar que los movimientos sociales y grupos activistas organizados - que responderían al concepto de insurgencia- son aquellos que desestiman, critican o simplemente invalidan los sistemas tradicionales de participación ciudadana - con lo que provocan una ruptura con el Estado—, y los declaran, en el lenguaje de Miraftab (2018), como los espacios convidados para la incidencia pública. Más aun, cuestionan y no se sienten parte de la categoría de ciudadanos.

Esta ruptura con la institucionalidad está respaldada por las nuevas cuestiones epistemológicas y ontológicas desarrolladas en la academia que precisamente critican lo institucional y hegemónico. Esto es lo que se denomina la decolonialidad del conocimiento occidental o epistemologías del sur (De Sousa Santos, 2010). Según este autor:

En el campo del conocimiento, se concede a la ciencia moderna el monopolio de la distinción universal entre lo verdadero y lo falso, en detrimento de dos cuerpos alternativos de conocimiento: la filosofía y la teología [...] conocimientos populares, laicos, plebeyos, campesinos o indígenas al otro lado de la línea. Desaparecen como conocimientos relevantes o conmensurables porque se encuentran más allá de la verdad y de la falsedad. (De Sousa Santos, 2010) 
Esta decolonialidad del saber recupera lo local y los saberes situados en la definición de lo que es bueno para un territorio, con lo que cuestiona la planificación tradicional.

La iraní Faranak Miraftab (2018) alude a razones estructurales de las políticas públicas. En su texto «Insurgencia, planificación y la perspectiva de un urbanismo humano», señala que estamos hoy en un momento de crisis, una crisis global que no es solamente una crisis del capitalismo, sino también de la planificación como profesión. La autora invita a repensar la planificación e imaginar — al igual que Imilan — prácticas de descolonización que hagan posible un urbanismo humano (Mansilla e Imilan, 2020). La planificación se está enfrentando con su propia crisis de identidad y legitimidad, una crisis que emerge de su esquizofrenia profesional, razón por la que su propia imagen e idea de la profesión no encajan.

Según Svirsky (2010), el Estado-nación utiliza su «poder métrico» para axiomatizar la política, mientras simultáneamente reprime experiencias de activistas que simplemente se niegan a alinearse con lo dado de la política formal. Un ejemplo de esto se puede ver en la hostilidad de los Estados occidentales hacia organizaciones como Wikileaks o el movimiento por los derechos de los animales, cada uno de los cuales está inmerso en actos de ciudadanía que actualizan rupturas. Esas nuevas escenas y actos están constantemente en riesgo de ser apropiados por esta ciencia real de la política, que les impone un modelo que canaliza la participación ciudadana según reglas y conceptos establecidos.

Activismos que buscan solo garantizar el funcionamiento de la democracia representativa son esencialmente activismos de esclavos; viven en la seguridad y su impacto y potencial se espera que sea absorbido, sin llevar el sistema a nuevas estructuras de resonancia. (Svirsky, 2010)

Para Miraftab (2018), hablar de prácticas insurgentes significa reconocer el abanico de prácticas existentes que van más allá de las que son sancionadas por el Estado y los poderes corporativos. Al descolonizar la imaginación, se provocaría la irrupción de movimientos anticoloniales y anticapitalistas de larga duración. Según ella, es ahí donde se encontrarán la inspiración, los valores y los principios orientadores para prácticas que pueden promover un futuro y un urbanismo más humanos.

La propuesta de Miratftab (2018) refiere a que los planificadores profesionales se encuentran generalmente al servicio del bien privado y no del bien común, lo que queda expresado en la tensión integración social versus expulsión (gentrificación, injusticia espacial). Esto deriva en una crisis de legitimidad de la planificación, lo que obliga a que muchos ciudadanos en el mundo tomen por cuenta propia sus respectivas ciudades. Se requiere, según la autora, una nueva imaginación, un nuevo léxico, un nuevo repertorio de prácticas y una comprensión distinta de justicia. 
A las denominadas prácticas insurgentes las considera como un tipo diferente de planificaciones, una planificación alternativa «hecha a la medida» y que tiene lugar entre comunidades subordinadas, sean asentamientos informales y municipios en excolonias o comunidades desfavorecidas. Según Miratftab, la falencia de la planificación inclusiva liberal nos fuerza a repensar los parámetros epistemológicos y ontológicos de las teorizaciones y prácticas de planificación. En este sentido, establece que es necesario brindar un reconocimiento de las prácticas ciudadanas como formas de planificación. James Holston (2007) desarrolla incluso el concepto de ciudadanía insurgente, cuando los pobladores presionan al sistema que finalmente los legitima, en una especie de acuerdo jurídico tácito entre unos y otros actores.

Pero ¿cómo son estas prácticas? Miraftab (2018) establece otra tensión, esta vez respecto de la participación ciudadana, que considera como «espacios de acción invitados» que deben modificarse por «espacios inventados» que entreguen reconocimiento urgente de formas de opresión autodeterminadas y basadas en grupos.

De esta manera, la planificación debe hacerse desde una acción directa colectiva de los ciudadanos, transitando de la democracia representativa a la democracia participativa. Las prácticas insurgentes y la planificación insurgente no buscan la inclusión a través de una mejor representación (sea de especialistas o de políticos), sino que buscan la inclusión autodeterminada, en la cual los derechos de las personas son reales y practicados. La preocupación principal es por las prácticas y no por sus actores (planificadores profesionales). El activismo que solo busca garantizar los mecanismos de la democracia representativa constituye lo que Svirsky (2010) llama «activismo esclavo» (planificación inclusiva a través de la participación ciudadana).

En opinión de este último, el poder subversivo de la potencia política es así contenido por esta estrategia reductora; la sociedad civil se convierte en el principal territorio de este encarcelamiento, asistido por una falsa ecuación de participación con política desafiante (Svirsky, 2010).

\section{Consideraciones finales}

Tal como señalan Iracheta Cenecorta e Iracheta Carroll, la acción pública ocurre por la coordinación de la acción colectiva y no por la subordinación de la ciudadanía al aparato gubernamental. Aquí encontramos una primera lección. El alicaído Estado-nación (al menos hasta antes de la pandemia) debe reconocer nuevas formas de organización social y política, que a su vez sean capaces de innovar en sus prácticas y salir de cierto estado de subordinación, evitando la instrumentalización. Los nuevos movimientos, si bien son atemporales y con patrimonio difuso - a diferencia de sus pares reconocidos en la ley-, pueden vincularse en beneficio de sus comunidades. Por otra parte, 
toda política pública debe ajustarse, bajo el principio de descentralización, a las necesidades territoriales y locales, para evitar la universalización de sus métodos y concepciones. Más aún en el caso de un Estado de tipo unitario como el chileno.

En definitiva, la participación política en sus expresiones electorales y ciudadanas ha disminuido en calidad e incidencia, aunque los rankings internacionales no lo reflejen. Tal como se ha revisado en este ensayo, la cuestión de la participación de la sociedad en las políticas públicas es una cuestión transversal del máximo interés. Hablamos de participación social considerando su acepción más amplia que la de participación ciudadana, ya que muchos de los grupos activistas o de tomas de terrenos cuestionan las categorías tradicionales de ciudadanía porque no se sienten incluidos en el sistema, como señala Holston (2007).

Los mecanismos de participación diseñados mediante la ley por el Estado chileno no representan más que esfuerzos desesperados de este y sus administradores tecnócratas por compartir poder y reducir su cierto sentimiento de culpa ante la injusticia social y territorial. Será tarea de los nuevos planificadores territoriales o urbanos incluir mecanismos deliberativos que permitan la construcción de una democracia participativa y no representativa o directa en ambos extremos.

La paradoja chilena demuestra que, en un sistema político representativo, una alta densidad normativa no asegura la inclusión de los grupos y demandas sociales, ya que está en crisis la legitimidad del sistema.

Por otra parte, las políticas públicas en materia de participación deberán ser capaces de salir de su ortodoxia metodológica y de requisitos si es que quieren incluir a los que buscan por fuera del sistema respuestas a sus demandas históricas. Los datos indican que el problema de la gobernanza no pasa entonces por el número de organizaciones constituidas, sino más bien por su incidencia efectiva en espacios de poder. En este sentido, la institucionalidad de las políticas públicas debe replantearse las formas de vinculación con las nuevas demandas sociales y ciudadanas, más que enfocarse en perfeccionar sistemas representativos de dudosa efectividad real.

Sin embargo, se abren nuevas interrogantes que permitirán definir con más exactitud el problema planteado en este ensayo: ¿Son los actuales dispositivos y mecanismos institucionales suficientes y pertinentes para recoger las demandas? En última instancia, ¿debe seguir el Estado intercediendo o son los propios vecinos los llamados a incidir directamente en los asuntos públicos que les corresponden? Este es el futuro camino de la participación ciudadana si es que se pretende evitar en el gobierno un estado de anarquía que lo aleje de los estándares esperables de gobernanza. 


\section{Referencias bibliográficas}

Arriagada Luco, C. (2017). La investigación sobre segregación socioespacial en Chile: Estado del conocimiento, metodologías de estudio y propuestas territoriales. Ponencia presentada en el 31. ${ }^{\circ}$ Congreso ALAS, Montevideo.

Castells, M. (2005). Globalización, desarrollo y democracia: Chile en el contexto mundial. Santiago de Chile: Fondo de Cultura Económica.

Castillo Couve, M. J. (2014). Competencias de los pobladores: Potencial de innovación para la política habitacional chilena. Revista INVI, 29(81), 79-112.

Chile. (1976). Decreto 458: DFL 458. Aprueba Ley General de Urbanismo y Construcciones. Recuperado de https://www.bcn.cl/leychile/navegar?idNorma=13560.

Chile. (1993). Ley 19.253: Establece normas sobre protección, fomento y desarrollo de los indígenas y crea la Corporación Nacional de Desarrollo Indígena. Recuperado de https://www.bcn.cl/leychile/navegar?idNorma=30620.

Chile. (1994). Ley 19.300: Aprueba ley sobre bases generales del medio ambiente. Recuperado de https://www.bcn.cl/leychile/navegar?idNorma=30667.

Chile. (1997). Decreto 58: Fija texto refundido, coordinado y sistematizado de la Ley 19.418 sobre Juntas de Vecinos y Demás Organizaciones Comunitarias. Recuperado de https://www.bcn.cl/leychile/navegar?idNorma $=70040$.

Chile. (2006). DFL 1: Fija texto refundido, coordinado y sistematizado de la Ley 18.695 Orgánica Constitucional sobre Municipalidades. Recuperado de https://www. bcn.cl/leychile/navegar?idNorma=251693.

Chile. (2008). Ley 20.285, sobre acceso a la información pública. Recuperado de https://www.bcn.cl/leychile/navegar?idNorma=276363.

Chile. (2011). Ley 20.500, sobre asociaciones y participación ciudadana en la gestión pública. Recuperado de https://www.bcn.cl/leychile/navegar?idNorma= 1023143.

Chile. (2018a). Ley 21.073, que regula la elección de gobernadores regionales y realiza adecuaciones a diversos cuerpos legales. Recuperado de https://www.bcn.cl/leychile/navegar?idNorma $=1115503$.

Chile. (2018b). Ley 21.074, de fortalecimiento de la regionalización en el país. Recuperado de https://www.bcn.cl/leychile/navegar?idNorma=1115064.

Comisión Económica para América Latina y el Caribe. (2016). Horizontes 2030: La igualdad en el centro del desarrollo sostenible. Santiago de Chile: CEPAL.

De Sousa Santos, B. (2010). Para descolonizar Occidente: Más allá del pensamiento abismal. Buenos Aires: Consejo Latinoamericano de Ciencias Sociales. (Perspectivas).

The Economist Intelligence Unit Limited. (2021). Democracy Index 2020: In sickness and in health? Recuperado de https://www.eiu.com/n/campaigns/democracy-index2020/\#mktoForm_anchor. 
Ferrero, M. (2010). Gobernando (en) un mundo en globalización: Una mirada conceptual a la gobernanza desde las relaciones internacionales. En L. E. Concepción Montiel y P. Moctezuma Hernández (coords.), Gobernanza global y democracia (pp. 147-170). México: Universidad Autónoma de Baja California.

Hernández Bonivento, J. (2018). La forma y la esencia: Efectos de las leyes de transparencia, acceso a la información y participación ciudadana en la gobernanza democrática local en Chile. Revista de Gestión Publica, 7(2), 143-169.

Herrmann, M. G., y van Klaveren, A. (2016). Disminución de la participación de la población en organizaciones sociales durante los últimos trece años en Chile e implicaciones para la construcción de una política de planificación urbana más participativa. EURE (Santiago), 42(125), 175-203. https://dx.doi.org/10.4067/S025071612016000100008.

Holston, J. (2007). Insurgent citizenship: Disjunctions of democracy and modernity in Brazil. Princeton: Princeton University Press.

Iracheta Cenecorta, A., e Iracheta Carroll, J. (2014). Evaluación de los fondos metropolitano y regional del Gobierno Federal Mexicano. México: CIDE. Recuperado de http://clear-la.cide.edu/sites/default/files/Evaluaci\%C3\%B3n\%20de\%20los\%20Fondos\%20Metropolitano\%20y\%20Regional_Iracheta\%20e\%20Iracheta.pdf.

Irarrázaval, I., y Streeter, P. (2020). Mapa de las organizaciones de la sociedad civil 2020. Santiago: Centro de Políticas. Recuperado de https://www.sociedadenaccion.cl/ wp-content//uploads/2020/07/Mapa-OSC-2020_Sociedad-en-Acci\%C3\%B3n.pdf.

Mansilla Quiñones, P., e Imilan Ojeda, W. (2020). Colonialidad del poder, desarrollo urbano y desposesión mapuche: Urbanización de tierras mapuches en la Araucanía chilena. Scripta Nova, 24(630).

Maya, J., Alanís, H., Ferrusca, F., y Sánchez, P. (2019). Análisis del concepto de gobernanza territorial desde el enfoque del desarrollo urbano. Estado, Gobierno y Gestión Pública, (31), 175-210.

Miraftab, F. (2018). Insurgencia, planificación y la perspectiva de un urbanismo humano. Territorios, (38). https://doi.org/10.12804/revistas.urosario.edu.co/territorios/a.6507.

Murphy, E. (2014). In and out of the margins urban land seizures and homeownership in Santiago Chile. En B. Fischer, B. McCann y J. Auyero (eds.), Book cities from scratch: Poverty and informality in urban Latin America (pp.68-101). Durham: Duke University Press.

Portal Martínez, J. M. (2016). Control interno e integridad: Elementos necesarios para la gobernanza pública. El Cotidiano, (198), 7-13. Recuperado de https://www.redalyc.org/articulo.oa?id=325/32546809002. 
Programa de las Naciones Unidas para el Desarrollo. (2017). Diagnóstico sobre la participación electoral en Chile. Proyecto fomentando la participación electoral en Chile: Por una ciudadanía más involucrada y participativa. Santiago de Chile: PNUD.

Sánchez, R. (ed.) (2019). La bonanza de los recursos naturales para el desarrollo: Dilemas de gobernanza. Santiago de Chile: CEPAL. (Libros de la CEPAL, 157).

Subirats, J., Knoepfel, P., Larrue, C., y Varonne, F. (2008). Análisis y gestión de políticas públicas. Barcelona: Ariel.

Svirsky, M. G. (2010). Introduction: Beyond the royal science of politics. Deleuze Studies, $4(\mathrm{~S} 1), 1-6$. 